\title{
STRESS-RELAXATION IN INCOMPRESSIBLE ELASTIC MATERIALS AT CONSTANT DEFORMATION*
}

\author{
BY \\ R. S. RIVLIN \\ Brown University
}

1. Introduction. The mechanics of the finite deformation of incompressible isotropic ideal elastic materials has received a considerable amount of attention in recent years ${ }^{1}$. In the present paper, we shall discuss the mechanics of the finite deformation of incompressible isotropic elastic materials which behave substantially as ideal elastic materials when subjected to deformations during a relatively short time interval (less than some time $T$, say,) but, if held in that state of deformation for times much greater than $T$, exhibit relaxation of the deforming forces. Such a material will be called a stressrelaxing elastic material. It will be shown that many of the results of the theory of the finite deformation of incompressible isotropic ideal elastic materials apply, with slight modification, to stress-relaxing elastic materials.

2. The stress in a stress-relaxing elastic material. We assume that a body of an isotropic stress-relaxing elastic material is subjected, at zero time, to a deformation in which points of the body initially at $X_{i}$, in the rectangular Cartesian coordinate system $x_{i}$, move to $x_{i}$ in the same coordinate system, so that

$$
x_{i}=x_{i}\left(X_{i}\right),
$$

where $x_{i}$ is independent of the time $t$, for $t>0$. We assume that the stress components $\sigma_{i j}$ in the coordinate system $x_{i}$, at a point of the material initially at $X_{i}$, are singlevalued functions of the nine gradients $\partial x_{i} / \partial X_{i}$ and of the time $t$; i.e.

$$
\sigma_{i i}=f_{i j}\left(\partial x_{p} / \partial X_{a}, t\right) \text {. }
$$

Since the material is assumed to be isotropic in its undeformed state, the functional form of the dependence of $\sigma_{i j}$ on $\partial x_{p} / \partial X_{q}$ and $t$ must be independent of the choice of the rectangular Cartesian coordinate system $x_{i}$. Now, suppose $x_{i}^{*}$ is a rectangular Cartesian coordinate system related to $x_{i}$ by

$$
x_{i}^{*}=a_{i j} x_{i}, \quad \text { where } \quad a_{i j} a_{k i}=\delta_{i k} .
$$

Then, if $X_{i}^{*}$ are the coordinates in the coordinate system $x_{i}^{*}$ of a point which is at $X_{i}$ in the coordinate system $x_{i}$, we have

$$
X_{i}^{*}=a_{i j} X_{i}
$$

If $\sigma_{i j}^{*}$ are the components of the stress at time $t$ in the coordinate system $x_{i}^{*}$, we have

$$
\sigma_{i i}^{*}=a_{i k} a_{i l} \sigma_{k l}=f_{i i}\left(\partial x_{p}^{*} / \partial X_{a}^{*}, t\right) .
$$

*Manuscript received April 19, 1955. The results contained in this paper were obtained in the course of work on a project sponsored by the Armstrong Cork Company. The author is indebted to Dr. G. W. Scott, Jr. and Dr. J. T. Bergen for their encouragement and to the Armstrong Cork Company for permission to publish the work.

'For reviews of the subject see C. Truesdell [1] and Green and Zerna [2]. 
From (2.5), it can be shown, in a manner similar to that adopted in the theory of the finite deformation of perfectly elastic materials (see, for example, Rivlin and Ericksen [3, Sec. 13]), that $\sigma_{i j}$ must be expressible in terms of $\partial x_{k} / \partial X_{\imath}$ through the components of a symmetric Cartesian tensor, which has components $C_{p q}$ in the coordinate system $x_{i}$ defined by

$$
C_{p e}=\frac{\partial x_{p}}{\partial X_{k}} \frac{\partial x_{q}}{\partial X_{k}}
$$

and that $\sigma_{i j}$ must be a single-valued function of $t$ and the components $C_{p a}$ of this tensor. If it is assumed that $\sigma_{i j}$ is expressible as a polynomial in the nine quantities $\partial x_{p} / \partial X_{a}$, with coefficients which are single-valued functions of $t$, then it follows that it must be expressible as a polynomial in the six independent components $C_{p a}$ of the tensor defined by (2.6), with coefficients which are single-valued functions of $t$.

If we represent the functional dependence of $\sigma_{i j}$ on $C_{p q}$ and $t$ by

$$
\sigma_{i j}=F_{i j}\left(C_{p q}, t\right)
$$

then, in view of the relations (2.5), we must have

$$
F_{i j}\left(C_{p a}^{*}, t\right)=a_{i k} a_{i l} F_{k l}\left(C_{p a}, t\right),
$$

where $C_{p q}^{*}$ is defined by

$$
C_{p a}^{*}=a_{p k} a_{a l} C_{k l}=\frac{\partial x_{p}^{*}}{\partial X_{k}^{*}} \frac{\partial x_{a}^{*}}{\partial X_{k}^{*}} .
$$

Employing the notation $\mathrm{d}=\left\|\sigma_{i j}\right\|$ and $\mathrm{C}=\left\|C_{i i}\right\|$, Eqs. (2.7) and (2.8) imply that $\boldsymbol{\sigma}$ is an isotropic matrix function of $\mathbf{C}$.

It can be shown, in a manner similar to that adopted by Rivlin and Ericksen [3, Sec. 39] in the case when $\delta$ is an isotropic matrix function of $C$ and the elements of $\delta$ do not depend on $t$, that a linear relation of the form

$$
\boldsymbol{\delta}=\alpha_{0} \mathrm{I}+\alpha_{1} \mathrm{C}+\alpha_{2} \mathrm{C}^{2},
$$

exists between $\delta, \mathbf{C}, \mathbf{C}^{2}$ and the unit matrix I. In this relation, $\alpha_{0}, \alpha_{1}$ and $\alpha_{2}$ are singlevalued functions of $t$ and the scalar invariants $\operatorname{tr} \mathbf{C}, \operatorname{tr} \mathbf{C}^{2}$ and $\operatorname{tr} \mathbf{C}^{3}$. If the elements of $\delta$ are expressible as polynomials ${ }^{2}$ in the elements of $\mathrm{C}$, then $\alpha_{0}, \alpha_{1}$ and $\alpha_{2}$ are expressible as polynomials in $\operatorname{tr} \mathbf{C}, \operatorname{tr} \mathbf{C}^{2}$ and $\operatorname{tr} \mathbf{C}^{3}$, with coefficients which are single-valued functions of $t$.

If the material is incompressible, then the stress is undetermined to the extent of an arbitrary hydrostatic pressure $p$ (say). We must then replace Eq. (2.7) by

$$
\sigma_{i j}=F_{i j}\left(C_{p a}, t\right)-p \delta_{i j} .
$$

The constancy of volume of an element in the deformation is expressible by

$$
\operatorname{det} C_{p q}=1 \text {, }
$$

which leads to the relation

$$
\operatorname{det} \mathbf{C}=\frac{1}{6}\left[2 \operatorname{tr} \mathbf{C}^{3}+(\operatorname{tr} \mathbf{C})^{3}-3 \operatorname{tr} \mathbf{C} \operatorname{tr} \mathbf{C}^{2}\right]=1 .
$$

${ }^{2}$ See also Reiner $[4,5]$ and Prager [6]. 
In a manner similar to that employed in arriving at Eq. (2.10) from (2.7), we obtain, from (2.11),

$$
\mathbf{d}=\alpha_{0} \mathbf{I}+\alpha_{1} \mathbf{C}+\alpha_{2} \mathbf{C}^{2}-p \mathbf{I},
$$

where $\alpha_{0}, \alpha_{1}$ and $\alpha_{2}$ are single-valued functions of $t$ and the scalar invariants $\operatorname{tr} \mathbf{C}, \operatorname{tr} \mathbf{C}^{2}$ and $\operatorname{tr} \mathrm{C}^{3}$. Since the relation (2.13) is satisfied $\alpha_{0}, \alpha_{1}$ and $\alpha_{2}$ may be expressed as singlevalued functions of $t$ and the scalar invariants $\operatorname{tr} \mathbf{C}$ and $\operatorname{tr} \mathbf{C}^{2}$. Also, since $p$ is arbitrary, we may re-write (2.14) as

$$
\delta=\alpha_{1} \mathbf{C}+\alpha_{2} \mathbf{C}^{2}-p I,
$$

where $\alpha_{1}$ and $\alpha_{2}$ are single-valued functions of $t$ and the scalar invariants $\operatorname{tr} \mathbf{C}$ and $\operatorname{tr} \mathbf{C}^{2}$

If, in (2.11), $F_{i}$ is a polynomial in the quantities $C_{p o}$ with coefficients which are single-valued functions of $t$, then, in (2.14), $\alpha_{0}, \alpha_{1}$ and $\alpha_{2}$ are polynomials in tr C and $\operatorname{tr} \mathrm{C}^{2}$ with coefficients which are single-valued functions of $t$. Therefore, in (2.15), $\alpha_{1}$ and $\alpha_{2}$ are polynomials in tr $\mathbf{C}$ and tr $\mathbf{C}^{2}$ with coefficients which are single-valued functions of $t$.

3. Comparison with the theory of finite elastic deformations. For a perfectly elastic isotropic material, subjected to a deformation described by (2.1), the stress matrix $\delta$ is given by

$$
\boldsymbol{\delta}=\alpha_{0} \mathbf{I}+\alpha_{1} \mathbf{C}+\alpha_{2} \mathbf{C}^{2},
$$

where $\alpha_{0}, \alpha_{1}$ and $\alpha_{2}$ are polynomials in the scalar invariants $I_{1}, I_{2}$ and $I_{3}$ defined by

$$
\begin{gathered}
I_{1}=\operatorname{tr} \mathbf{C}, \quad I_{2}=\frac{1}{2}\left[(\operatorname{tr} \mathbf{C})^{2}-\operatorname{tr} \mathbf{C}^{2}\right], \\
I_{3}=\operatorname{det} \mathbf{C}=\frac{1}{6}\left[2 \operatorname{tr} \mathbf{C}^{3}+(\operatorname{tr} \mathbf{C})^{3}-3 \operatorname{tr} \mathbf{C} \operatorname{tr} \mathbf{C}^{2}\right] .
\end{gathered}
$$

$\alpha_{0}, \alpha_{1}$ and $\alpha_{2}$ are therefore expressible as polynomials in $\operatorname{tr} \mathbf{C}, \operatorname{tr} \mathbf{C}^{2}$ and $\operatorname{tr} \mathbf{C}^{3}$. The coefficients in these polynomials are not, in this case, functions of time as in the case of the stress-relaxing elastic material. They are, however, derivable from a strain-energy function $W$, which is expressible as a polynomial in $I_{1}, I_{2}$ and $I_{3}$, by means of the relations

$$
\alpha_{0}=2 I_{3}^{1 / 2} \frac{\partial W}{\partial I_{3}}, \quad \alpha_{1}=\frac{2}{I_{3}^{1 / 2}}\left(\frac{\partial W}{\partial I_{1}}+I_{1} \frac{\partial W}{\partial I_{2}}\right)
$$

and

$$
\alpha_{2}=-\frac{2}{I_{3}^{1 / 2}} \frac{\partial W}{\partial I_{2}}
$$

If the perfectly elastic isotropic material is incompressible, then the stress matrix $\boldsymbol{d}$ is given by

$$
\boldsymbol{\delta}=\alpha_{1} \mathrm{C}+\alpha_{2} \mathrm{C}-p \mathrm{I},
$$

where $p$ is an arbitrary hydrostatic pressure and $\alpha_{1}$ and $\alpha_{2}$ are now expressible as polynomials in $I_{1}$ and $I_{2}$ only, while $I_{3}=1$. Since $I_{3}=1$, it is seen from (3.2) that $\alpha_{1}$ and $\alpha_{2}$ must be expressible as polynomials in $\operatorname{tr} \mathrm{C}$ and $\operatorname{tr} \mathrm{C}^{2}$ only. $\alpha_{1}$ and $\alpha_{2}$ are derivable from 
a strain-energy function $W$, which is expressible as a polynomial in $I_{1}$ and $I_{2}$ only, by means of the relations

$$
\alpha_{1}=2\left(\frac{\partial W}{\partial I_{1}}+I_{1} \frac{\partial W}{\partial I_{2}}\right) \quad \text { and } \quad \alpha_{2}=-2 \frac{\partial W}{\partial I_{2}} .
$$

Since the stress-relaxing elastic materials considered in Sec. 2 are assumed to behave substantially as perfectly elastic materials, if the deformation is carried out in a sufficiently small time interval, $\alpha_{0}, \alpha_{1}$ and $\alpha_{2}$ in the expression (2.10) for the stress matrix must be expressible in the form (3.3) when $t=0$. Similarly, in the case of an incompressible stress-relaxing elastic material, $\alpha_{1}$ and $\alpha_{2}$ in the expression (2.14) for the stress matrix must be expressible in the form (3.5) when $t=0$.

However, after stress relaxation has taken place, the $\alpha$ 's need no longer be expressible in terms of a strain-energy function. It is noted that, even in this case, the expressison for the stress matrix for the stress-relaxing and perfectly elastic materials are identical apart from the fact that for the stress-relaxing material the $\alpha$ 's depend on time and are not necessarily expressible in terms of a strain-energy function. Also, we shall assume, since this involves no added complication, that the $\alpha$ 's are not necessarily expressible as polynomials in the scalar invariants of $\mathbf{C}$, but are, more generally, single-valued functions of these.

4. The applied forces for a specified deformation. The body forces $f_{i}$ per unit mass of material and surface tractions $F_{i}$ per unit area of surface measured in the deformed state, which must be applied to a body in order to maintain the stress distribution $\sigma_{i j}$ in it, are given by

$$
\begin{gathered}
\frac{\partial \sigma_{i j}}{\partial x_{i}}+\rho f_{i}=0, \\
F_{i}=\sigma_{i i} l_{i},
\end{gathered}
$$

where $\rho$ is the density of the material of the body in its deformed state, $l_{i}$ are the directioncosines of the normal to the surface of the body in its deformed state and it is assumed that the body is held in a constant state of deformation.

Equations (4.1) apply to both the stress-relaxing and perfectly elastic materials in both the compressible and incompressible cases. In the theory of the finite deformation of perfectly elastic materials, they have been used to calculate the forces necessary to support a number of simple types of deformation in the incompressible case using the stress-deformation relation (3.4), in which $\alpha_{1}$ and $\alpha_{2}$ are given by (3.5). In most of these problems no explicit use is made of the fact that $\alpha_{1}$ and $\alpha_{2}$ depend on the scalar invariants $I_{1}$ and $I_{2}$. In these cases we can take over the results of the theory of the finite deformation of perfectly elastic incompressible isotropic materials and apply them to the stress-relaxing elastic materials discussed in Sec. 2 by replacing $\partial W / \partial I_{1}$ and $\partial W / \partial I_{2}$ by $\frac{1}{2}\left(\alpha_{1}+I_{1} \alpha_{2}\right)$ and $-\frac{1}{2} \alpha_{2}$ respectively.

We shall consider a few results in the theory of the finite deformation of perfectly elastic incompressible isotropic materials which, with this modification, apply also to stress-relaxing isotropic incompressible materials.

(a) Simple extension. For a uniform rod of perfectly elastic incompressible isotropic material, subjected to a tensile force $F$, the force $F$ is given (Rivlin [7]) in terms of the extension ratio $\lambda$ by the relation 


$$
F=2 A\left(\lambda-\frac{1}{\lambda^{2}}\right)\left(\frac{\partial W}{\partial I_{1}}+\frac{1}{\lambda} \frac{\partial W}{\partial I_{2}}\right),
$$

where $A$ is the cross-sectional area of the rod in its deformed state and $I_{1}$ and $I_{2}$ are given by

$$
I_{1}=\lambda^{2}+\frac{2}{\lambda} \quad \text { and } \quad I_{2}=2 \lambda+\frac{1}{\lambda^{2}} .
$$

Making the substitutions $\frac{1}{2}\left(\alpha_{1}+I_{1} \alpha_{2}\right)$ and $-\frac{1}{2} \alpha_{2}$, where $\alpha_{1}$ and $\alpha_{2}$ are functions of $I_{1}, I_{2}$ and $t$, for $\partial W / \partial I_{1}$ and $\partial W / \partial I_{2}$ respectively, we obtain, for the incompressible, isotropic, stress-relaxing elastic material,

$$
F=A\left(\lambda-\frac{1}{\lambda^{2}}\right)\left[\alpha_{1}+\left(I_{1}-\frac{1}{\lambda}\right) \alpha_{2}\right],
$$

where $I_{1}$ and $I_{2}$ are still given by (4.3). Substituting for $I_{1}$ in (4.4), we obtain

$$
F=A\left(\lambda-\frac{1}{\lambda^{2}}\right)\left[\alpha_{1}+\left(\lambda^{2}+\frac{1}{\lambda}\right) \alpha_{2}\right] \text {. }
$$

(b) Simultaneous simple extension and torsion of a circular rod or tube. If a tube of isotropic incompressible perfectly elastic material is subjected simultaneously to a simple extension of extension ratio $\lambda$ and torsion of amount $\psi$, the force system which must be applied in order to maintain the deformation consists of (i) azimuthal surface tractions applied to the plane ends of the tube of magnitude $\theta$ per unit area measured in the deformed state of the tube; (ii) normal surface tractions applied to the plane ends of the tube of magnitude $Z$ per unit area measured in the deformed state of the tube; (iii) normal surface tractions applied to the inner curved surface of the tube of magnitude $P$ per unit area measured in the deformed state of the tube. $\psi$ is defined as the angle of twist per unit length of the rod in its extended state. $\theta, Z$ and $P$ are given (Rivlin [8]), at a point distant $r$ from the axis of the tube in its undeformed state, by

$$
\begin{gathered}
\Theta=2 \psi \lambda^{3 / 2} r\left(\frac{\partial W}{\partial I_{1}}+\frac{1}{\lambda} \frac{\partial W}{\partial I_{2}}\right) \\
Z=2\left[\left(\lambda^{2}-\frac{1}{\lambda}\right)\left(\frac{\partial W}{\partial I_{1}}+\frac{1}{\lambda} \frac{\partial W}{\partial I_{2}}\right)-\psi^{2} r^{2} \frac{\partial W}{\partial I_{2}}\right]+2 \lambda \psi^{2} \int_{a}^{r} r \frac{\partial W}{\partial I_{1}} d r \\
P=-2 \psi^{2} \lambda \int_{b}^{e} r \frac{\partial W}{\partial I_{1}} d r .
\end{gathered}
$$

In these equations $I_{1}$ and $I_{2}$ are given by

$$
I_{1}=\lambda^{2}+\frac{2}{\lambda}+\psi^{2} \lambda r^{2} \quad \text { and } \quad I_{2}=\frac{1}{\lambda^{2}}+2 \lambda+\psi^{2} r^{2}
$$

and $a$ and $b$ are the external and internal radii of the tube in its undeformed state.

The azimuthal surface tractions $\theta$ are statically equivalent to a couple $M$ given by

$$
M=4 \pi \psi \int_{b}^{a} r^{3}\left(\frac{\partial W}{\partial I_{1}}+\frac{1}{\lambda} \frac{\partial W}{\partial I_{2}}\right) d r
$$


and the normal surface tractions $Z$ to a longitudinal force $N$ given by

$$
N=4 \pi\left(\lambda-\frac{1}{\lambda^{2}}\right) \int_{b}^{a}\left(\frac{\partial W}{\partial I_{1}}+\frac{1}{\lambda} \frac{\partial W}{\partial I_{2}}\right) r d r-2 \pi \psi^{2} \int_{b}^{a} r^{3}\left(\frac{\partial W}{\partial I_{1}}+\frac{2}{\lambda} \frac{\partial W}{\partial I_{2}}\right) d r .
$$

By taking $b=0$ in the first two of Eqs. (4.6) and in Eqs. (4.7) to (4.9), we obtain the results appropriate to a rod of circular cross-section and radius $a$ in its undeformed state.

Replacing $\partial W / \partial I_{1}$ and $\partial W / \partial I_{2}$ by $\frac{1}{2}\left(\alpha_{1}+I_{1} \alpha_{2}\right)$ and $-\frac{1}{2} \alpha_{2}$ respectively in Eqs. (4.6) to (4.9) and substituting the expression (4.7) for $I_{1}$, we obtain the forces which must be applied to a tube of our stress-relaxing elastic material in order to maintain in it a state of simultaneous simple extension and torsion. Thus, we have

$$
\begin{aligned}
\Theta= & \psi \lambda^{3 / 2} r\left[\alpha_{1}+\left(\lambda^{2}+\frac{1}{\lambda}+\psi^{2} \lambda r^{2}\right) \alpha_{2}\right] \\
Z=\left(\lambda^{2}-\frac{1}{\lambda}\right)\left[\alpha_{1}+\left(\lambda^{2}+\frac{1}{\lambda}+\psi^{2} \lambda r^{2}\right) \alpha_{2}\right]+ & \psi^{2} r^{2} \alpha_{2} \\
& +\lambda \psi^{2} \int_{a}^{r} r\left[\alpha_{1}+\left(\lambda^{2}+\frac{2}{\lambda}+\psi^{2} \lambda r^{2}\right) \alpha_{2}\right] d r
\end{aligned}
$$

and

$$
P=-\psi^{2} \lambda \int_{b}^{a} r\left[\alpha_{1}+\left(\lambda^{2}+\frac{2}{\lambda}+\psi^{2} \lambda r^{2}\right) \alpha_{2}\right] d r
$$

while $M$ and $N$ are given by

$$
\begin{aligned}
& M=2 \pi \psi \int_{b}^{a} r^{3}\left[\alpha_{1}+\left(\lambda^{2}+\frac{1}{\lambda}+\psi^{2} \lambda r^{2}\right) \alpha_{2}\right] d r \\
& N=2 \pi\left(\lambda-\frac{1}{\lambda^{2}}\right) \int_{b}^{a} r\left[\alpha_{1}+\left(\lambda^{2}+\frac{2}{\lambda}+\psi^{2} \lambda r^{2}\right) \alpha_{2}\right] d r \\
& -\pi \psi^{2} \int_{b}^{a} r^{3}\left[\alpha_{1}+\left(\lambda^{2}+\psi^{2} \lambda r^{2}\right) \alpha_{2}\right] d r .
\end{aligned}
$$

We again obtain the results appropriate to a rod of circular cross-section by taking $b=0$ in the expressions for $\theta$ and $Z$ given in (4.10) and in (4.11).

(c) Small torsion superposed on simple extension for a uniform rod of arbitrary crosssection. It has been shown by Green and Shield [9] ${ }^{3}$ that if a rod of isotropic incompressible perfectly elastic material, of arbitrary uniform cross-section, is subjected to an extension of extension ratio $\lambda$ and to an infinitesimal torsion of amount $\psi$, measured per unit length of rod in its extended state, then the tensile force $N$ and torsional couple $M$ which must be applied in order to maintain the rod in this state of deformation are given by

'See also [2]. The argument of Green and Shield given in [9] has been modified in detail by Gent and Rivlin [10]. 


$$
\begin{aligned}
& N=2 A\left(\frac{\partial W}{\partial I_{1}}+\frac{1}{\lambda} \frac{\partial W}{\partial I_{2}}\right)\left(\lambda-\frac{1}{\lambda^{2}}\right), \\
& M=2 \psi\left(\frac{\partial W}{\partial I_{1}}+\frac{1}{\lambda} \frac{\partial W}{\partial I_{2}}\right)\left\{I-\frac{1}{\lambda^{3}}(I-S)\right\},
\end{aligned}
$$

where $I_{1}$ and $I_{2}$ are given by

$$
I_{1}=\lambda^{2}+\frac{2}{\lambda} \quad \text { and } \quad I_{2}=\frac{1}{\lambda^{2}}+2 \lambda
$$

$A$ denotes the cross-sectional area of the rod in its undeformed state, $I$ denotes the moment of inertia of the cross-section of the rod, in its undeformed state, about the axis of torsion and $S$ denotes the geometrical torsional rigidity of the unextended rod when subjected to a small twist.

From Eqs. (4.12) and (4.13), we see that for infinitesimal amounts of torsion superposed on a state of simple extension, we have the following relation which is independent of the precise form of the strain-energy funstion

$$
\frac{N}{M / \psi}=\frac{\left(\lambda-1 / \lambda^{2}\right) A}{I-\left(I-S^{3}\right) / \lambda^{3}}
$$

Again, following through the analysis of Green and Shield, but employing the expressions (2.14) for the stress in our stress-relaxing elastic material, we can obtain expressions identical with (4.12), in which $\partial W / \partial I_{1}$ and $\partial W / \partial I_{2}$ are replaced by $\frac{1}{2}\left(\alpha_{1}+I_{1} \alpha_{2}\right)$ and $-\frac{1}{2} \alpha_{2}$ respectively, where $\alpha_{1}$ and $\alpha_{2}$ are functions of $I_{1}, I_{2}$ and $t$. These expressions give the tensile force and torsional couple which are required to support a simultaneous simple extension and infinitesimal torsion in our stress-relaxing elastic material. Again, from these expressions we can obtain the expression (4.14), which is thus seen to be valid also for a stress-relaxing elastic material if the extensional and torsional deformations are simultaneously applied in times small compared with those in which appreciable relaxation of stress can take place in the material, the rod is held subsequently in this state of deformation and the tensile force $N$ and torsional couple $M$ are measured simultaneously at an arbitrary time after the deformation is produced.

\section{REFERENCES}

1. C. Truesdell, J. Rat'l. Mech. \& Anal. 2, 125 (1952); 3, 593 (1953)

2. A. E. Green and W. Zerna, Theoretical elasticity, The Clarendon Press, Oxford, 1954

3. R. S. Rivlin and J. L. Ericksen, J. Rat'l. Mech. \& Anal. 4, 323 (1955)

4. M. Reiner, Amer. J. Math. 67, 350 (1945)

5. M. Reiner, Amer. J. Math. 70, 433 (1948)

6. W. Prager, J. Appl. Phys. 16, 837 (1945)

7. R. S. Rivlin, Phil. Trans. A 241, 379 (1948)

8. R. S. Rivlin, Phil. Trans. A 242, 173 (1949)

9. A. E. Green and R. T. Shield, Phil. Trans. A 244, 47 (1951)

10. A. N. Gent and R. S. Rivlin, Proc. Phys. Soc. B 65, 645 (1952) 